\title{
Metodologia para geração de sinais de arco elétrico para otimização de cenários de medições
} Methodology for radio signal generation in the study of high voltage circuit breakers

Nieremberg J. P. de Lyra Ramos ${ }^{1}$, Alexandre Jean Rene Serres ${ }^{2}$, Edson Guedes da Costa ${ }^{3}$, Alfredo Gomes Neto ${ }^{4}$

de Lyra Ramos, N.J.P; Rene Serres, A.J; Guedes da Costa, E; Gomes Neto, A. Metodologia para geração de sinais de arco elétrico para otimização de cenários de medições. Tecnología en Marcha. Vol. 34, especial. ALTAE. Diciembre 2021. Pág 245-255.

doi) https://doi.org/10.18845/tm.v34i7.6048

1 Universidade Federal de Campina Grande, Departamento de Engenharia Elétrica, Programa de Pós-graduação em Engenharia Elétrica.

Correo electrónico: nieremberg.ramos@ee.ufcg.edu.br

2 Universidade Federal de Campina Grande, Departamento de Engenharia Elétrica, Programa de Pós-graduação em Engenharia Elétrica. Correo electrónico: alexandreserres@dee.ufcg.edu.br

3 Universidade Federal de Campina Grande, Departamento de Engenharia Elétrica, Programa de Pós-graduação em Engenharia Elétrica. Correo electrónico: edson@dee.ufcg.edu.br

4 Instituto Federal de Educação, Ciência e Tecnologia da Paraíba, Programa de Pós-graduação em Engenharia Elétrica.

Correo electrónico: alfredogomes@ieee.com 


\section{Palavras chave}

Radiometria; diagnóstico; disjuntores; arco elétrico; geração de sinais.

\section{Resumo}

As subestações concentram equipamentos essenciais para o funcionamento das redes de distribuição de energia elétrica. O diagnóstico do estado de degradação dos equipamentos é muito importante, em especial dos disjuntores que são os responsáveis por seccionar a rede. Uma técnica que tem se destacado é o diagnóstico de disjuntores a partir da análise do sinal irradiado pelo arco elétrico, que ocorre na comutação de estados nos disjuntores. Essa técnica compreende a captação do sinal irradiado, seu armazenamento em formato digital, e análise a partir de informações obtidas. Um problema que essa técnica enfrenta é a otimização dos cenários de medições, pois para isso é necessário estar em uma subestação, onde as comutações são realizadas sem controle dos pesquisadores, ou possuir disjuntores em laboratório. Porém, em laboratório existem limitações quanto aos tipos de disjuntores pela impossibilidade de ter vários disjuntores em laboratório. A metodologia de otimização proposta neste artigo visa a reprodução de sinais radiométricos de arcos elétricos, previamente adquiridos, em subestações ou em laboratório, tornando possível simular em ambiente experimental disjuntores diversos, em diferentes estágios de degradação. Dessa forma, é possível otimizar os cenários de medições, variando distâncias e posicionamentos das antenas e de outros elementos, realizando medições sucessivas para obter a melhor configuração de medição.

\section{Keywords}

Radiometry; diagnostics; circuit breakers; electric arc; signal generation.

\section{Abstract}

The substations concentrate essential equipment for the operation of electric energy distribution networks. The diagnosis of the state of degradation of equipment is very important, especially of the circuit breakers that are responsible for sectioning the network. One technique that has stood out is the diagnosis of circuit breakers based on the analysis of the signal radiated by the electric arc, which occurs in the switching of states in circuit breakers. This technique comprises capturing the radiated signal, storing it in digital format, and analyzing it from the information obtained. One problem that this technique faces is the optimization of measurement scenarios, because for this it is necessary to be in a substation, where the changes are carried out without the researchers' control, or to have circuit breakers in the laboratory. However, in the laboratory there are limitations regarding the types of circuit breakers due to the impossibility of having several circuit breakers in the laboratory. The optimization methodology proposed in this article aims at reproducing radiometric signals from electrical arcs, previously acquired, in substations or in a laboratory, making it possible to simulate in an experimental environment different circuit breakers, in different stages of degradation. In this way, it is possible to optimize the measurement scenarios, varying distances and positions of antennas and other elements, performing successive measurements to obtain the best measurement configuration. 


\section{Introdução}

Desde a revolução industrial, quando a energia elétrica se mostrou um fator determinante para o desenvolvimento da humanidade, a demanda por esse tipo de energia tem aumentado gradualmente a cada ano diante do aumento populacional e do aumento de equipamentos e dispositivos conectados aos sistemas elétricos de potência [1]. Com esse aumento de demanda, os sistemas elétricos precisam se adequar e com isso também há uma maior necessidade de manutenções, pois o sistema é composto por diversos equipamentos, além dos condutores, para garantir o transporte confiável entre os centros geradores e as áreas consumidoras. Dentre estes equipamentos, pode-se citar os transformadores de potência, isoladores, para-raios, transformadores de instrumentos, chaves, disjuntores de alta tensão entre outros [2].

Os disjuntores são elementos essenciais para a operação dos sistemas elétricos de forma segura, pois precisam realizar a comutação de circuitos, abrindo ou fechando os seus contatos sempre que necessário. As manutenções preventivas destes disjuntores são atividades de complexidade operativa significativa, uma vez que, nos métodos tradicionais, é necessário interromper o sistema, e em alguns envolve a remoção e desmontagem dos equipamentos para diagnosticar seus estados de conservação. Estima-se que aproximadamente $45 \%$ das falhas em sistemas elétricos estão relacionadas com o estado de conservação dos disjuntores [3].

Para reduzir os impactos das manutenções dos disjuntores, formas de diagnósticos não invasivas têm sido cada vez mais exploradas. Nestas formas de manutenção não se faz necessária a remoção ou abertura dos disjuntores para a verificação do seu estado de conservação. Dentre as técnicas atualmente utilizadas, uma que se destaca é a análise radiométrica do estado de conservação dos disjuntores, pois faz uso sinais eletromagnéticos irradiados pelo disjuntor no momento da sua comutação de estados [4]-[6].

O fenômeno que possibilita essa análise é a formação do arco elétrico, que é um fenômeno físico transitório, que ocorre entre os terminais dos disjuntores quando eles estão em movimento de aproximação ou se afastamento. Na formação desse arco elétrico é irradiado um sinal eletromagnético com características de ignição, extinção, duração e amplitude. A partir da análise das características desse sinal é possível estimar o estado de conservação dos contatos dos disjuntores [7]-[9].

Um problema enfrentado por essa técnica radiométrica de análise de disjuntores está na preparação das configurações dos cenários de medições, pois antes de ir às subestações realizar os diagnósticos é necessário otimizar os componentes do conjunto de medições, como posicionamento de antenas, distanciamento, influência de outros elementos alheios ao cenário de medições. No entanto, para cada otimização é necessário um acionamento dos disjuntores, para assim poder verificar os parâmetros de medições necessários. No Brasil, o controle das subestações, e consequentemente os comandos de comutação dos disjuntores, são dados pelo operador nacional do sistema e assim sendo, não há domínio dos pesquisadores sobre o momento em que haverá a comutação dos disjuntores. Em laboratório é necessário ter os disjuntores conectados a altas tensões ou altas correntes, o que envolve riscos, e se mostra um cenário limitado, pois para realizar medições de disjuntores com diferentes meios de extinção é necessário ter diversos disjuntores e caracterizar contatos com diferentes estados de conservação.

Visando solucionar este problema, esta pesquisa apresenta uma nova metodologia, onde os sinais são gerados através de um gerador vetorial de sinais, utilizando dados previamente armazenados em medições anteriores em subestações ou laboratórios, utilizando diferentes pares de contatos, com diversos estados de conservação. Assim os pesquisadores terão 
controle sobre o momento em que precisam que o sinal seja gerado e poderão analisar diferentes disjuntores, fazendo a mudança apenas do arquivo com o sinal carregado no gerador de sinais.

Este artigo está estruturado da seguinte forma: na segunda seção é apresentado um comparativo das técnicas tradicionais de diagnóstico de disjuntores e a técnica radiométrica; na terceira seção é apresentada a metodologia a ser implementada; na quarta seção são apresentados resultados preliminares da implementação da metodologia e por fim as considerações finais sobre a pesquisa.

\section{Comparativo de técnicas de diagnóstico}

Um dos equipamentos das subestações que estão sob constante supervisão e avaliação são os disjuntores de alta tensão. Como eles são os responsáveis pela segurança e proteção dos circuitos, precisam estar sempre em bom estado de funcionamento quando forem acionados. Nesse processo de manutenção, alguns métodos de diagnóstico foram desenvolvidos. A seguir serão brevemente apresentados alguns desses métodos e comparados ao método radiométrico.

\section{Resistência estática de contato}

Nessa forma de diagnóstico são feitas conexões aos terminais dos disjuntores, a fim de avaliar o fluxo de corrente entre os contatos, pois quando um par de contatos metálicos, mecanicamente independentes, é exposto a um fluxo de corrente elétrica, a resistência entre eles deverá ser a menor possível. Assim, nessa técnica são feitas quatro conexões ao disjuntor, aplicandoIhe uma corrente elétrica contínua a uma determinada tensão. Os contatos permanecem estáticos na posição fechado, depois é feita a aferição da queda de tensão no outro terminal, possibilitando a obtenção da resistência entre os contatos [10].

\section{Resistência dinâmica de contato}

Assim como ocorre na técnica da resistência estática de contato, os terminais são submetidos a um corrente contínua a uma determinada tensão. Quando os contatos do disjuntor estão fechados, a resistência tende a zero, e quando estão abertos tende a infinito, impedindo assim que haja fluxo de corrente entre os terminais. Essa técnica de medição é aplicada na abertura dos contatos, com a resistência seguindo de valores teóricos de zero a infinito. O processo inverso não é aplicado porque gera ruídos indesejados que acabam atrapalhando a medição [11]. Ao realizar a abertura dos contatos, os valores de tensão e corrente sofrerão alterações que serão registradas para posteriormente servirem de base para o cálculo da resistência dinâmica de contato, esses valores serão dispostos em um gráfico apresentando a curva de resistência, normalmente expressa em função do tempo de abertura ou da distância entre os contatos, possibilitando assim uma melhor análise do estado dos contatos do disjuntor. Mesmo em processo de afastamento ainda haverá fluxo de corrente devido à geração do arco elétrico. A figura 1 ilustra o leiaute de medições de RDC, utilizando um equipamento da marca Megger [12]. 


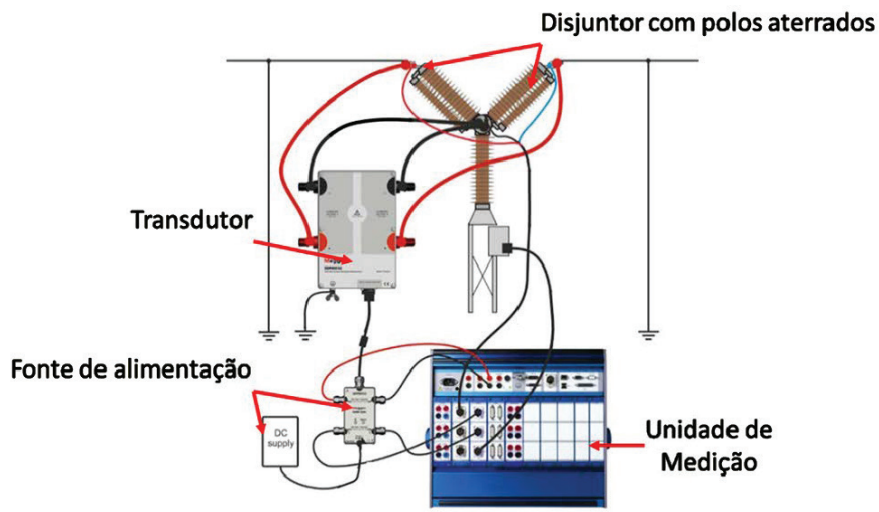

Figura 1. Exemplo de leiaute de medições de RDC em disjuntores de alta tensão. Fonte: [12].

A técnica radiométrica se mostra mais eficiente do que as técnicas de medição de resistência de contatos, tanto a estática como a dinâmica, porque utilizando radiometria não é necessário realizar conexões aos disjuntores, bastando apenas a captação do sinal radiométrico irradiado do disjuntor no momento de abertura.

\section{Análise termográfica}

No fluxo de correntes entre um par de contatos metálicos haverá uma resistência, mesmo que mínima, tendendo a zero, mas haverá, e essa resistência ocasionará a troca de energia elétrica em energia térmica, alterando assim a temperatura dos disjuntores. Quanto maior for a resistência entre os contatos, maior será o aumento dessa temperatura [13].

A termografia infravermelha é uma técnica de diagnóstico não invasiva que realiza a medição térmica do equipamento utilizando dispositivos optoeletrônicos, relacionando a radiação infravermelha com a temperatura superficial do equipamento [14].

A termografia infravermelha é uma forma de análise muito utilizada em ambiente industrial. Nesta técnica é feita a captação da radiação infravermelha do equipamento a uma determinada distância, identificando os níveis isotérmicos e determinando a temperatura do equipamento [15].

A figura 2 mostra um termovisor, equipamento utilizado para detectar padrões de calor no espectro do comprimento de onda infravermelho.

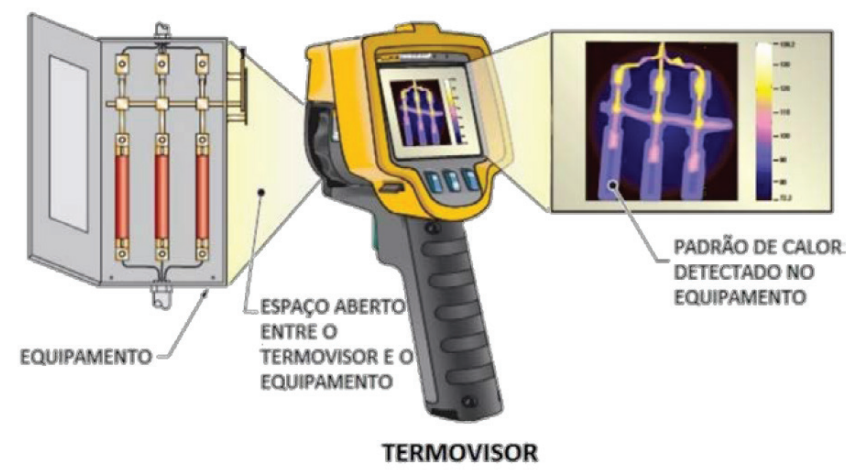

Figura 2. Imagem termográfica detectada através de termovisor. Fonte: [14]. 


\section{Análise radiométrica}

É uma técnica que tem se destacado recentemente, e ela se baseia na análise dos padrões dos sinais irradiados, na formação dos arcos elétricos, nas operações de abertura e fechamento dos contatos [16]-[18]. A vantagem desta técnica é a possibilidade de realizar a análise dos disjuntores em funcionamento, de forma não invasiva e sem a necessidade de remoção do aparelho. O que ainda tem se apresentado como fator limitador para essa técnica são os equipamentos necessários para a montagem do leiaute de medições para a aquisição dos sinais. Nos trabalhos mais recentes, encontrados na literatura, são utilizadas antenas ou acopladores magnéticos para a aquisição dos sinais radiométricos. Em seguida estes sinais são amostrados e discretizados para só então serem submetidos a um pós-processamento [4], [6], [19]-[22]. Na figura 3 é possível ver o leiaute de medições, com diferentes formas de aquisição do sinal. Em (a) é utilizado o acoplamento capacitivo, e em (b) é possível ver o leiaute de medições para a obtenção dos sinais irradiados pelo disjuntor utilizando uma antena acoplada ao osciloscópio.

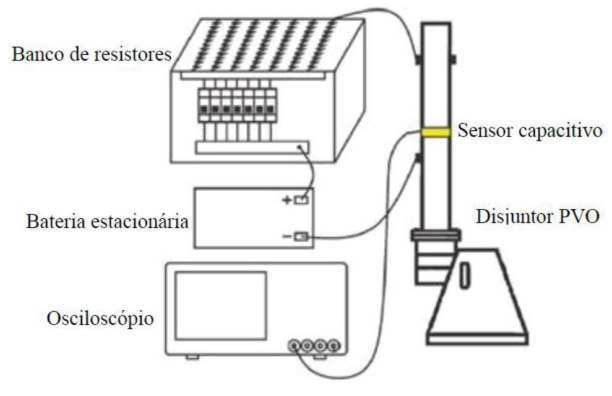

(a) Sensor capacitivo

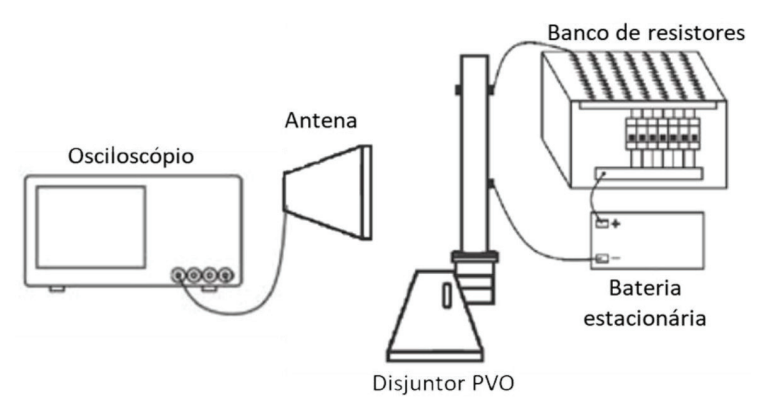

(b) Antena

Figura 3. Esquema de leiaute de medições com diferentes formas de aquisição do sinal. Fonte: [4], [6].

\section{Metodologia a ser implementada}

Inicialmente foram coletados sinais previamente adquiridos em outras pesquisas. Em seguida foram feitas análises do sinal para compreender a sua composição, para só então reproduzir os sinais em laboratório com o auxílio do gerador de sinais.

Para a reprodução do sinal foi utilizado o leiaute descrito no diagrama da figura 4, contendo um computador, um gerador de sinais, um analisador vetorial e um osciloscópio digital.

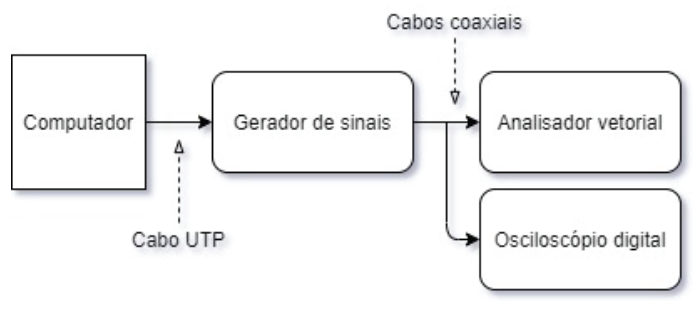

Figura 4. Diagrama de conexões do leiaute. 
Inicialmente, para validar a geração dos sinais, foram realizadas gerações utilizando sinais periódicos simples e pulsos quadrados para avaliar o seu comportamento com o osciloscópio, como pode ser visto na figura 5. Os sinais gerados inicialmente foram uma senoide, um pulso quadrado e um trem de pulsos. Os sinais medidos foram coerentes com os sinais gerados no Matlab, e a forma dos sinais no Matlab se assemelhavam à envoltória dos sinais gerados.

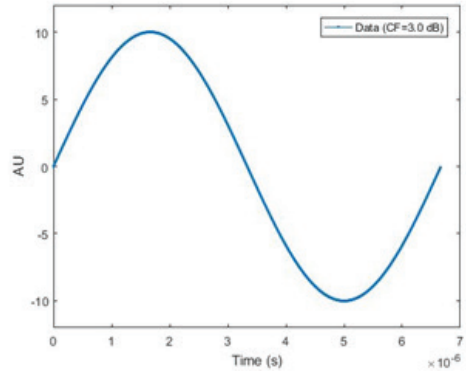

(a) Senoide no domínio do tempo

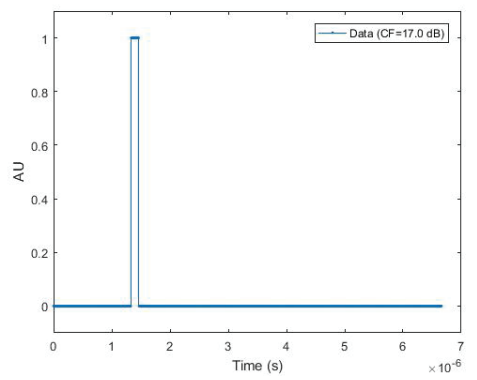

(c) Pulso quadrado no domínio do tempo

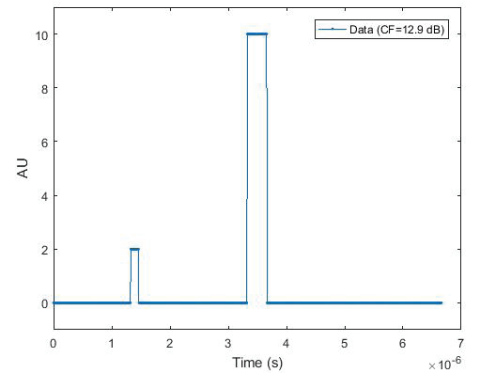

(e) Pulsos no domínio do tempo

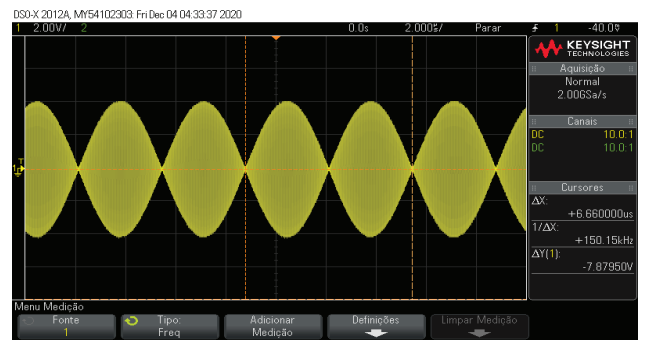

(b) Medição da senoide gerada

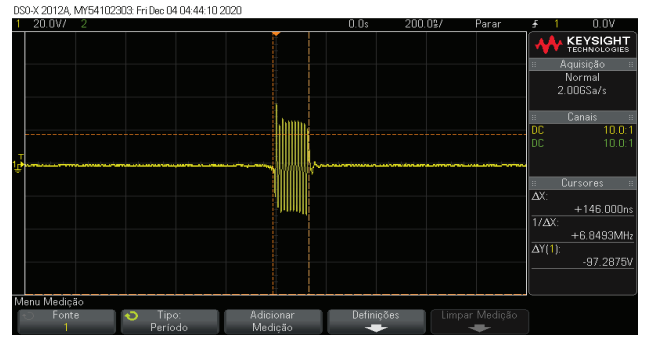

(d) Medição do pulso quadrado gerado

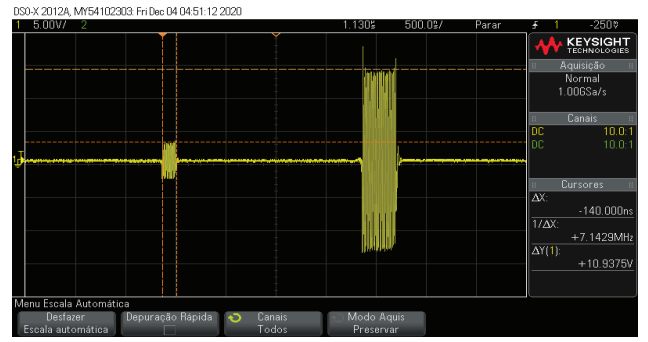

(f) Medição dos pulsos gerados

Figura 5. Sinais criados no Matlab, gerados no gerador de sinais e medidos no osciloscópio.

Sendo assim, observou-se que ao modular o sinal de banda básica sobre a portadora, o resultado obtido no osciloscópio foi coerente com o sinal carregado. A figura 6 mostra o leiaute de medições utilizado para os testes iniciais da metodologia de geração e medição dos sinais, ainda sem a utilização de antenas para a transmissão e recepção dos sinais. Nesta etapa o que importa é a validação da técnica implementada. 


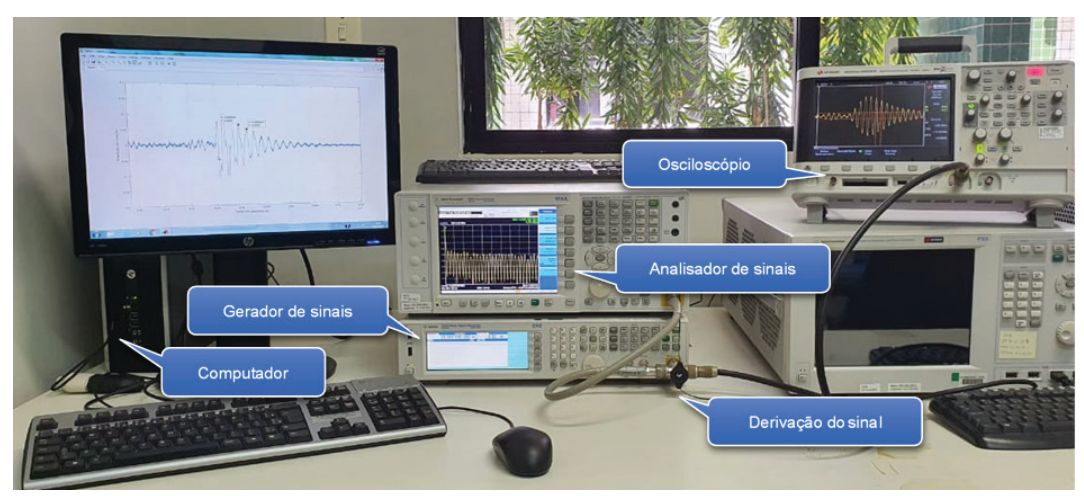

Figura 6. Leiaute de geração e medição de sinais.

Posteriormente foi montado o leiaute utilizando antenas para a transmissão e recepção do sinal, ao mesmo tempo que foi utilizada uma derivação para enviar o sinal para o osciloscópio também através do cabo, para que fosse possível a comparação dos resultados de medição, como pode ser visto na figura 7.

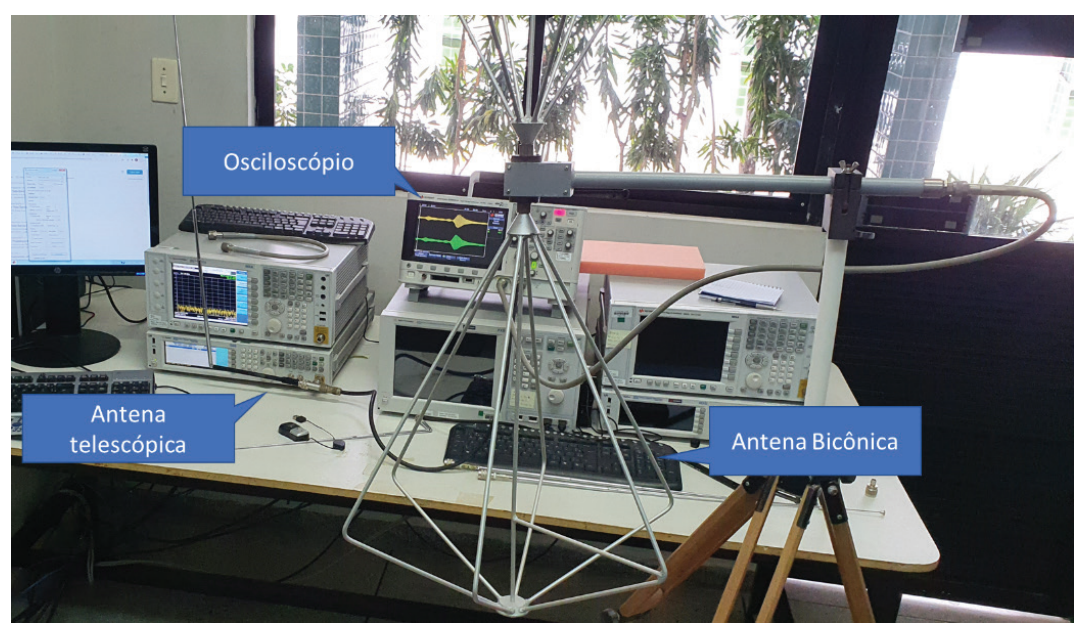

Figura 7 - Leiaute de geração e medição de sinais utilizando antenas.

\section{Resultados preliminares}

Após a validação da técnica de geração e medição dos sinais, outros sinais previamente adquiridos de disjuntores em laboratório foram utilizados para a geração e medição. Os sinais foram medidos tanto por antenas como pela transmissão do gerador ao osciloscópio através de cabos, na comparação dos sinais pode ser comprovado que há muita similaridade, como pode ser visto na figura 8.

Também foi feita a comparação do sinal recebido no osciloscópio com o sinal previamente armazenado, e verificou-se que as informações necessárias ao diagnóstico dos disjuntores estavam presentes no sinal gerado e que o mesmo era muito similar ao sinal previamente armazenado, como pode ser visto na comparação da figura 9 com a figura 10. 


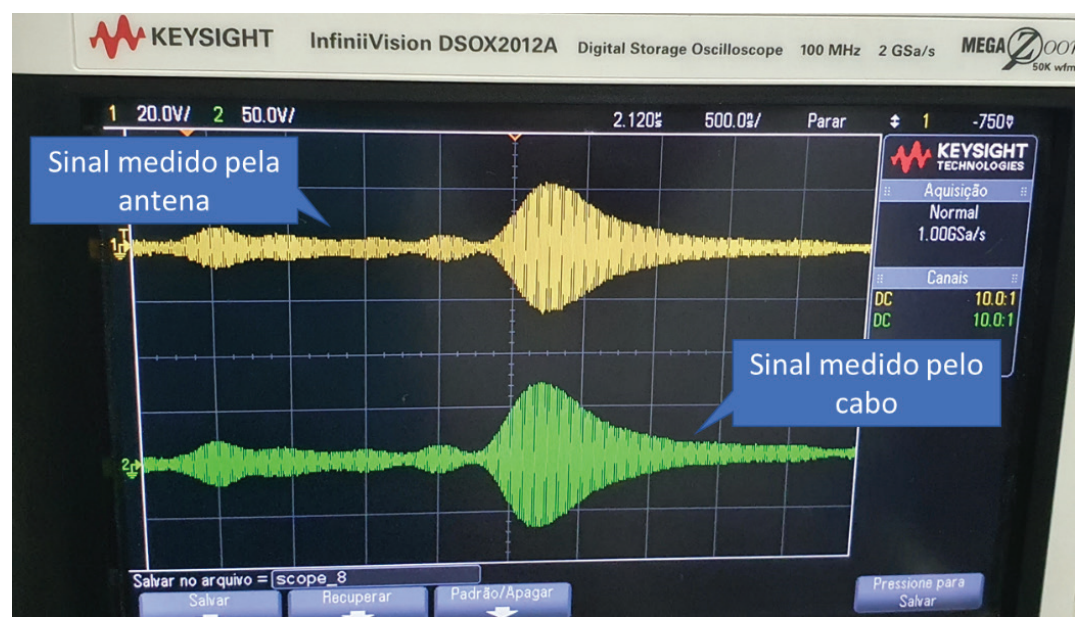

Figura 8 - Comparação de sinais recebidos no osciloscópio.

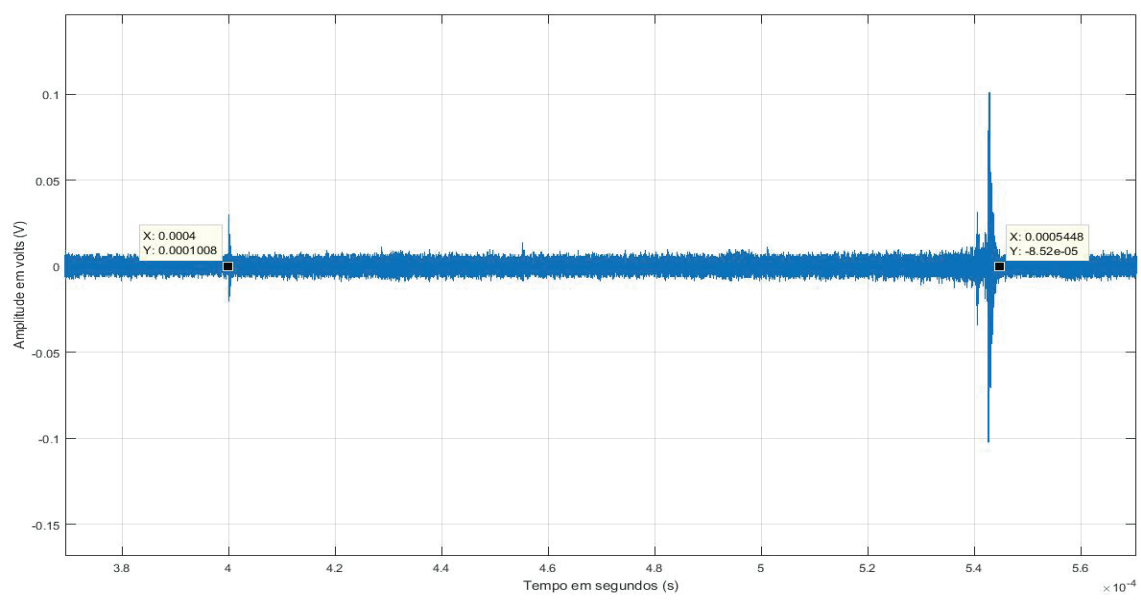

Figura 9. Sinal do arco elétrico armazenado.

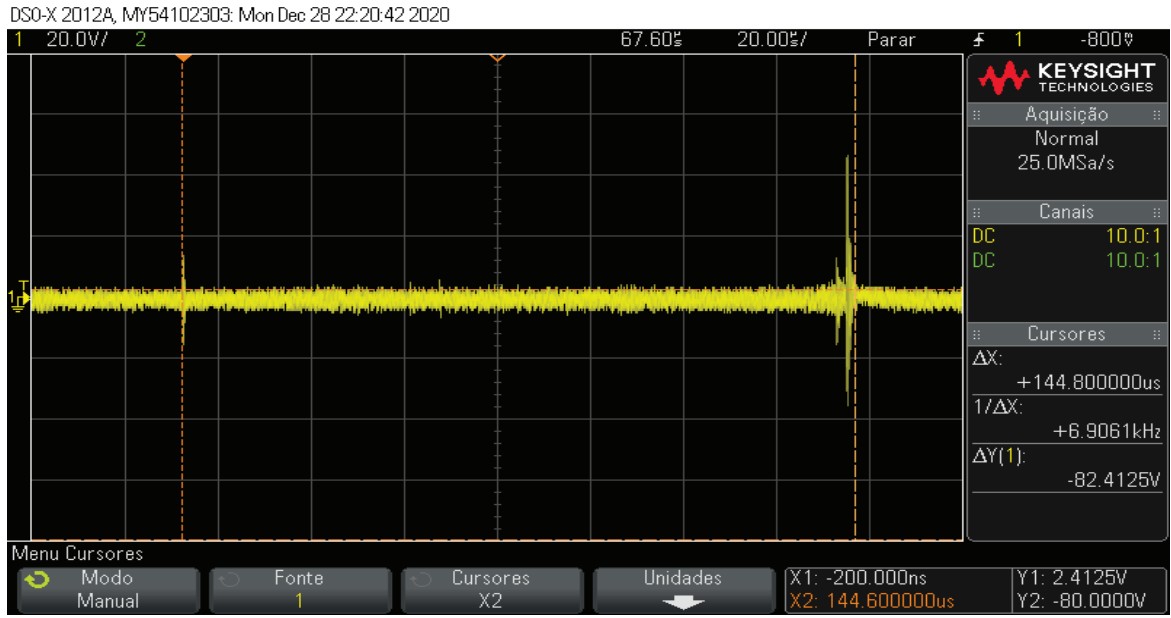

Figura 10. Sinal do arco elétrico gerado. 
Ao realizar uma comparação mais aproximada dos sinais é possível ver a similaridade deles, como pode ser visto na figura 11 e na figura 12. Eles não serão idênticos, mas carregam as mesmas informações de ignição, extinção e duração do arco elétrico, que são os parâmetros necessários à análise do estado de conservação dos contatos dos disjuntores.

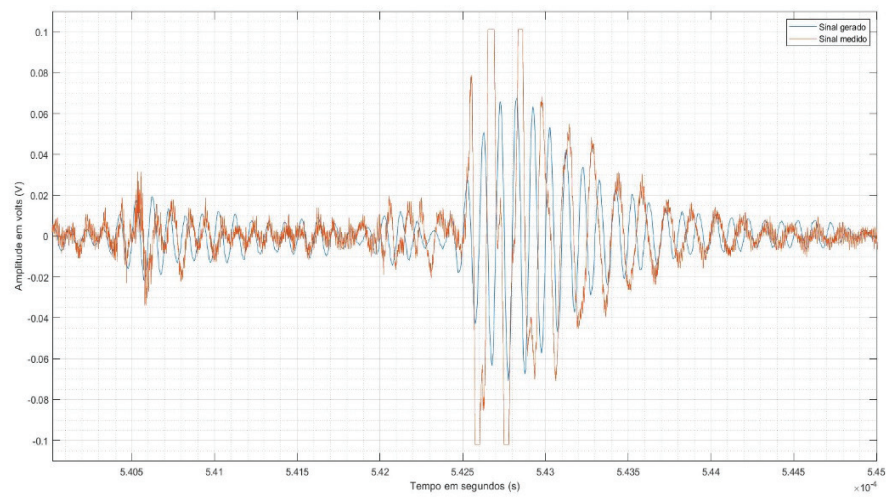

Figura 11. Comparação da região de ignição do arco elétrico.

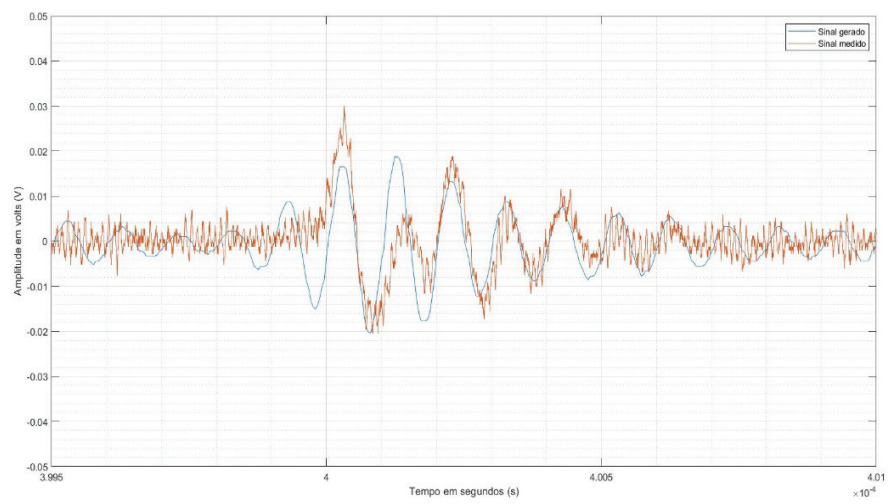

Figura 12. Comparação da região de extinção do arco elétrico.

\section{Considerações finais}

A metodologia proposta se mostra muito promissora para o auxílio dos pesquisadores na preparação dos leiautes de medições, pois foi possível verificar nos ensaios realizados que é viável reproduzir uma grande gama de sinais com fidelidade ao sinal original. Sendo assim, os comportamentos dos arcos elétricos em diferentes tipos de disjuntores poderão ser reproduzidos em laboratório, sem a necessidade de possuir diversos disjuntores.

A metodologia ainda requer avaliações para estabelecer os seus limites de aplicação, mas os indicadores são bastante promissores, pois cenários mais simples já podem ser reproduzidos.

\section{Referências}

[1] J. Goldemberg and O. Lucon, Energia, Meio Ambiente e Desenvolvimento, 3a Edição. São Paulo: EDUSP, 2012.

[2] J. D. MacDonald, Electric Power Substations Engineering, Third Edit. Boca Raton: CRC Press, 2016. 
[3] U. Katschinski, W. Olszewski, and T. Schaeffler, "A new generation of control and diagnostic techniques for gas insulated switchgear," in Proceedings of EMPD '98. 1998 International Conference on Energy Management and Power Delivery (Cat. No.98EX137), 1998, vol. 2, pp. 607-612, doi: 10.1109/EMPD.1998.702756.

[4] A. C. de Oliveira, "Método de monitoramento radiométrico para disjuntores de alta tensão," Universidade Federal de Campina Grande, 2019.

[5] A. C. de Oliveira, E. G. da Costa, A. J. R. Serres, H. F. dos S. Sousa, T. V. Ferreira, and H. N. de Santana, "Radiometric Monitoring Technique for High-Voltage Circuit Breakers," IEEE Trans. Power Deliv., vol. 34, no. 4, pp. 1656-1665, Aug. 2019, doi: 10.1109/TPWRD.2019.2917380.

[6] H. F. dos S. Sousa, "Desenvolvimento de técnica de detecção otimizada de sinais do arco elétrico para o monitoramento de disjuntores de alta tensão," Universidade Federal de Campina Grande, 2018.

[7] M. Chapman, "Radio-frequency-based determination of arcing duration in HVAC circuit breakers," in 18th International Conference and Exhibition on Electricity Distribution (CIRED 2005), 2005, vol. 2005, pp. v1-88-v1-88, doi: 10.1049/cp:20050964.

[8] G. Kuhlmann et al., "An analysis of the performance of power circuit breakers using the modelling of electric arc and a radiometric system," in 2017 International Symposium on Electromagnetic Compatibility - EMC EUROPE, Sep. 2017, pp. 1-6, doi: 10.1109/EMCEurope.2017.8094713.

[9] R. M. Harris, X. Hu, M. D. Judd, and P. J. Moore, "Detection and location of arcing faults in distribution networks using a non-contact approach," in 2012 IEEE International Power Modulator and High Voltage Conference (IPMHVC), Jun. 2012, pp. 583-586, doi: 10.1109/IPMHVC.2012.6518811.

[10] Megger, "Contact resistance test sets," 2020. https://megger.com/products/circuit-breaker-test-equipment/ contact-resistance-test-sets (accessed Feb. 13, 2020).

[11] M. Landry et al., "A New Measurement Method of the Dynamic Contact Resistance of HV Circuit Breakers," in 2006 IEEE/PES Transmission \& Distribution Conference and Exposition: Latin America, 2006, pp. 1-8, doi: 10.1109/TDCLA.2006.311501.

[12] Megger, "SDRM202 - Static/Dynamic Resistance MeasurementAccessory for TM1800/TM1700/EGIL," 2020. https://embed.widencdn.net/pdf/plus/megger/ouldce5uib/SDRM201_DS_en.pdf (accessed Feb. 03, 2020).

[13] H. C. V. Ness, Understanding Thermodynamics. New York: McGraw-Hill, 1983.

[14] Fluke, Introduction to Thermography - Principles. American Technical Publishers, 2009.

[15] A. P. Gebran, Manutenção e Operação de Equipamentos de Subestações: Série Tekne. Bookman Editora, 2014.

[16] J. Lopez-Roldan et al., "Development of non-intrusive monitoring for reactive switching of high voltage circuit breaker," Int. J. Electr. Power Energy Syst., vol. 61, pp. 219-228, 2014, doi: 10.1016/j.ijepes.2014.03.048.

[17] T. I. A. H. Mustafa et al., "An analysis of the performance of power circuit breakers using a non-invasive system," in 2016 IEEE/PES Transmission and Distribution Conference and Exposition (T\&D), May 2016, pp. 1-4, doi: 10.1109/TDC.2016.7519976.

[18] P. H. V. Rocha, E. G. Costa, A. R. Serres, G. V. R. Xavier, J. E. B. Peixoto, and R. L. Lins, "Inspection in overhead insulators through the analysis of the irradiated RF spectrum," Int. J. Electr. Power Energy Syst., vol. 113, pp. 355-361, Dec. 2019, doi: 10.1016/j.ijepes.2019.05.060.

[19] H. Ahmad and T. S. Kiong, "Trip Coil Signature Measurement and Analysis Techniques for Circuit Breaker," in 2016 7th International Conference on Intelligent Systems, Modelling and Simulation (ISMS), Jan. 2016, pp. 261-267, doi: 10.1109/ISMS.2016.28.

[20] M. Landry, O. Turcotte, and F. Brikci, "A Complete Strategy for Conducting Dynamic Contact Resistance Measurements on HV Circuit Breakers," IEEE Trans. Power Deliv., vol. 23, no. 2, pp. 710-716, Apr. 2008, doi: 10.1109/TPWRD.2008.917694.

[21] S. D. Meier, P. J. Moore, and P. F. Coventry, "Radiometric Timing of High-Voltage Circuit-Breaker Opening Operations," IEEE Trans. Power Deliv., vol. 26, no. 3, pp. 1411-1417, Jul. 2011, doi: 10.1109/ TPWRD.2011.2106225.

[22] T. I. Mustafa et al., "An analysis of the performance of $245 \mathrm{KV}$ circuit breakers applying the radiometric monitoring and the wavelet transform," in 2014 International Symposium on Electromagnetic Compatibility, Sep. 2014, pp. 1058-1062, doi: 10.1109/EMCEurope.2014.6931059. 